\title{
The IceTop Scintillator Upgrade
}

\section{The IceCube-Gen2 Collaboration ${ }^{\dagger}$}

$\dagger$ http://icecube.wisc.edu/collaboration/authors/icrc17_gen2

E-mail: samridha.kunwar@desy.de

The IceCube Neutrino Observatory at the South Pole probes the high-energy cosmic-ray sky by investigating the muonic and electromagnetic component of air showers measured with IceTop and the in-ice detector. However, more detailed measurements are needed to understand the astrophysics of the high-energy cosmic-ray sky. This, along with the need to mitigate the impact of snow accumulation on IceTop tanks, has given us impetus for further upgrades including scintillator and SiPM-readout-based stations. Prototype stations showcasing technological advances for the next generation in cosmic ray detection are currently under construction for deployment at South Pole in December 2017. We describe the physics and the current status of the project.

Corresponding authors: Thomas Huber ${ }^{1,3}$, John Kelley ${ }^{2}$, Samridha Kunwar ${ }^{* 3}$, Delia Tosi ${ }^{2}$

${ }^{1}$ Institut für Kernphysik, Karlsruhe Institute of Technology, D-76021 Karlsruhe, Germany

2 Dept. of Physics and Wisconsin IceCube Particle Astrophysics Center, University of Wisconsin, Madison, WI 53706, USA

${ }^{3}$ DESY, D-15738 Zeuthen, Germany

35th International Cosmic Ray Conference - ICRC2017

10-20 July, 2017

Bexco, Busan, Korea

* Speaker. 


\section{Introduction}

IceCube is a cubic-kilometer neutrino detector installed in the ice at the geographic South Pole [1] between depths of $1450 \mathrm{~m}$ and $2450 \mathrm{~m}$, which was completed in 2010 . Reconstruction of the direction, energy, and flavor of the neutrinos relies on the optical detection of Cherenkov radiation emitted by charged particles produced in the interactions of neutrinos in the surrounding ice or the nearby bedrock. Additionally, an array of surface detectors, IceTop, has also been deployed for cosmic ray studies in the PeV energy range [2] and to provide a partial veto of the down-going background of penetrating muons.

Accumulating snow cover of the IceTop tanks is continuously increasing the energy threshold for the detection of cosmic ray air showers [3]. The complex attenuation effects of the snow add systematic uncertainties to air shower measurements, particularly in the mass composition analysis. We have designed and proposed an upgrade to IceTop consisting of an homogeneously-spaced scintillator array with an areal coverage similar to IceTop. Currently we plan on deploying up to 37 scintillator stations over several years as shown in Fig 1. Each scintillator station comprises of seven scintillator panels communicating with a central hub as shown in Fig 2.

This upgrade is planned for two phases of deployment. Phase 1, marked in red in Fig. 1, will enable us to study the effect of attenuation on high-energy air showers. As stations are added during phase 2, marked in green in Fig. 1, the coverage will improve and the energy sensitivity will expand to include low-energy air showers.

\section{System Overview}

We are exploring different detector designs as prototype solutions for the upgrade. The system (Fig. 2) features the following building blocks:

- The scintillator panel subsystem. Extruded plastic scintillators are used in combination with wavelength-shifting fibers to collect and guide the light produced by an energetic particle crossing the scintillator. The fibers are read out by one light sensor. The detector is enclosed in a mechanical structure, designed to be light-tight and provide protection from harsh environmental conditions. 


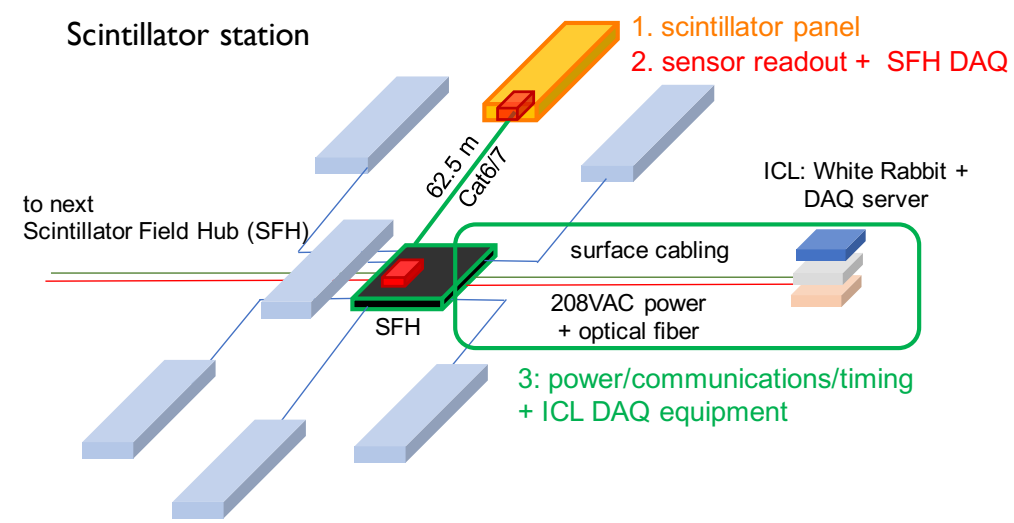

Figure 2: Scintillator station with 7 scintillator panels connected to a Field Hub DAQ featuring White Rabbit timing. The three building blocks as described in the text are highlighted.

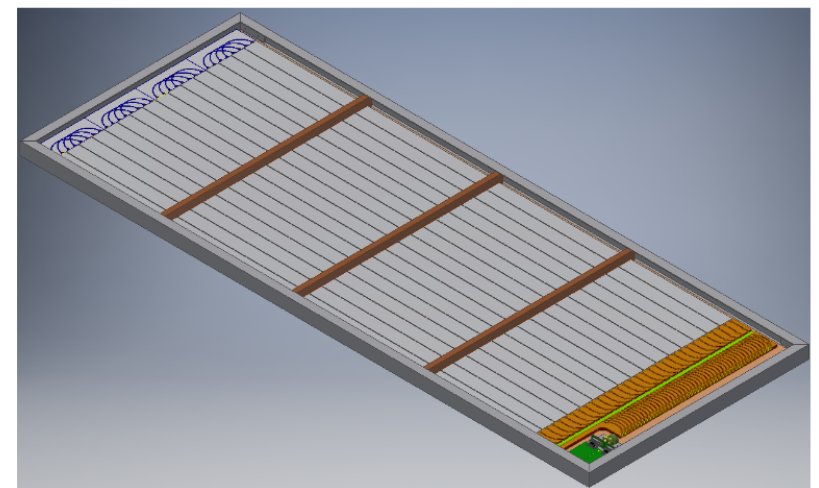
data acquisition system. equipment in the ICL. in more detail.

\section{The scintillator panel subsystem}

- The DAQ subsystem: the light sensor is read out and data are processed by a custom-designed

- The Scintillator Field Hub (SFH) subsystem includes data handling, power, and timing distribution between the IceCube Laboratory (ICL) and each scintillator station. It also includes

We have developed multiple realizations for each of the building blocks to explore different solutions in terms of costs and complexity. In the following sections we describe each subsystem

Figure 3: Left: Layout of the detector without top plate. The detector is placed in an aluminum frame to provide support. Right: optical fiber routing, plastic scintillator bars and a prototype of the Analog Readout Module for the IceTAXI Design (sec. 4.1), fully attached to the optical coupling of the detector. The SiPM is inside the cookie coupler which connects the optical fibers with the cookie board.

The detector design utilizes the low-cost customizable geometry of extruded scintillators simat K2K [4], the T2K near detector [5], MINERvA [6], and Auger Prime [7]. 
The unit detector is designed to have a total sensitive area of $1.5 \mathrm{~m}^{2}$ and a total weight of less than $50 \mathrm{~kg}$, to be easily transportable by two people. Each panel comprises 16 extruded plastic scintillator bars (produced on the FNAL/NICADD ${ }^{1}$ Extrusion Line), made of polystyrene with doping of $1 \%$ PPO and $0.03 \%$ POPOP and coated with a $0.25 \mathrm{~mm} \pm 0.13 \mathrm{~mm}$ thick layer of $\mathrm{TiO}_{2}$ reflector. Each bar is $1 \mathrm{~cm}$ thick, $5 \mathrm{~cm}$ wide and $1.875 \mathrm{~m}$ long and has two holes with a diameter of $2.5 \pm 0.2 \mathrm{~mm}$. Two Y-11(300) wavelength shifter fibers (produced by Kuraray ${ }^{2}$ ) are routed into the two holes of two bars, resulting in a bundle of 32 fiber ends which is then readout by a $6 \times 6 \mathrm{~mm}^{2}$ Silicon PhotoMultiplier (SiPM).

We are currently exploring the use of either $0.7 \mathrm{~mm}$ or $1 \mathrm{~mm}$ fiber, which offer different advantages in terms of cost and light yield. The light yield has been studied with a detailed GEANT4 [8] simulation of the apparatus, resulting in $82.3 \pm 23.9$ and $137.1 \pm 36.7$ photons absorbed in the SiPM for the $0.7 \mathrm{~mm}$ and $1 \mathrm{~mm}$ fiber respectively.

The fiber routing inherits features that were intensively studied for the AugerPrime upgrade of the Pierre Auger Observatory with the goal of optimizing sensitivity and uniformity. The bars are wrapped in opaque Velostat (ESD) material to ensure light-tightness. An outer aluminum shell and an aluminum frame provide support. A prototype panel is shown in Fig. 3.

Two types of coupling are being explored. In the first type, the bundled fibers are cast into a solid cookie with the fiber ends being cut and heated on a hot glass plate ensuring robustness and long term stability. The cookie is then glued into a PMMA (Polymethyl methacrylate) coupler along with the SiPM on a PCB - the cookie board. A $1 \mathrm{~mm}$ gap between the SiPM and the fiber ends is filled with optical glue (EJ-500 ${ }^{3}$ ) suited for low temperatures. This gap allows the illumination of every pixel on the SiPM and thereby increases the dynamic range, The second coupling makes use of a custom designed printed circuit board (PCB) that features holes of the same diameter as the fiber and are homogeneously spaced to guarantee illumination of the whole sensor active area. The fibers are threaded into the PCB and glued with a cold-rated epoxy. The bundle is then cut and polished with a slant cabochon lapidary polisher. The fiber bundle is then pressed against the SiPM surface with a spring loaded mechanism, with or without coupling gel.

\section{The DAQ subsystem}

\subsection{IceTAXI}

In this design, the SiPM is first connected to an analog readout module that comprises: the cookie board with analog and digital temperature sensors; an adapter board designed for mechanical stability that allows the cookie board and SiPM to be mounted inside the cookie; a readout board that houses the power supply for the SiPM and three different pre-amplifiers $(\times 1, \times 5, \times 10)$; and finally, a general purpose board that contains line drivers to transmit the analog signal from the scintillator panel to the SFH and a micro-controller for slow control (temperature, current, and voltage monitoring and control) via an RS485 connection.

\footnotetext{
${ }^{1}$ http://www.fnal.gov/facilities

${ }^{2}$ http: //kuraraypsf.jp

${ }^{3}$ http://www.ggg-tech.co.jp/maker/el jen/ej-500.html
} 
The IceTAXI DAQ is an FPGA + ARM embedded Linux system as shown in Fig. 4.1 that was developed for the TAXI project [9]. The original TAXI board supports 24 analog input channels in three blocks of eight channels each. A slightly modified version of the board, IceTAXI, with one block of eight channels was produced for the IceTop scin-

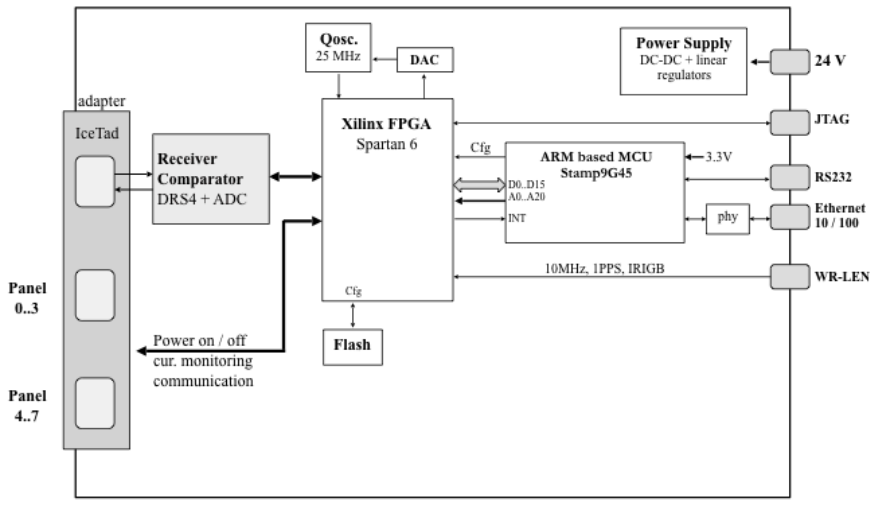

Figure 4: Layout of the IceTAXI Board. tillator array. Each channel is discriminated and the leading and trailing edges of the discriminator output are timestamped in a Spartan 6 FPGA with nanosecond precision. To achieve this, the output from the individual discriminators are interfaced with Serial-Input Parallel-Output (SIPO) Serializer Deserializer (SerDes) blocks in the FPGA with a 8:1 ratio at 950MHz. The subsequent 8-bit parallel output is then recorded at $118.75 \mathrm{MHz}$. In addition, input waveforms are recorded with a switched capacitor array and the integrated charge is determined online in the FPGA. The pulse time information and the charge-integrated or full waveform are then transferred to an ARM microcontroller unit running Linux that handles the data formatting and transmission to the IceCube Laboratory via a $1 \mathrm{~Gb}$ fiber link under a White Rabbit layer.

\section{2 $\mu \mathrm{DAQ}$}

The $\mu \mathrm{DAQ}$ is a small microprocessorbased DAQ board (Fig. 4.2) dedicated to a single sensor (SiPM connected to a scintillator panel) that includes power, timing and communications hub and spokes for medium remote connection $(\sim 60 \mathrm{~m})$. The microprocessor, along with inexpensive logic, captures sensor pulse start and stop with $\sim 1$ ns resolution. To achieve this, eight delayed inputs are used

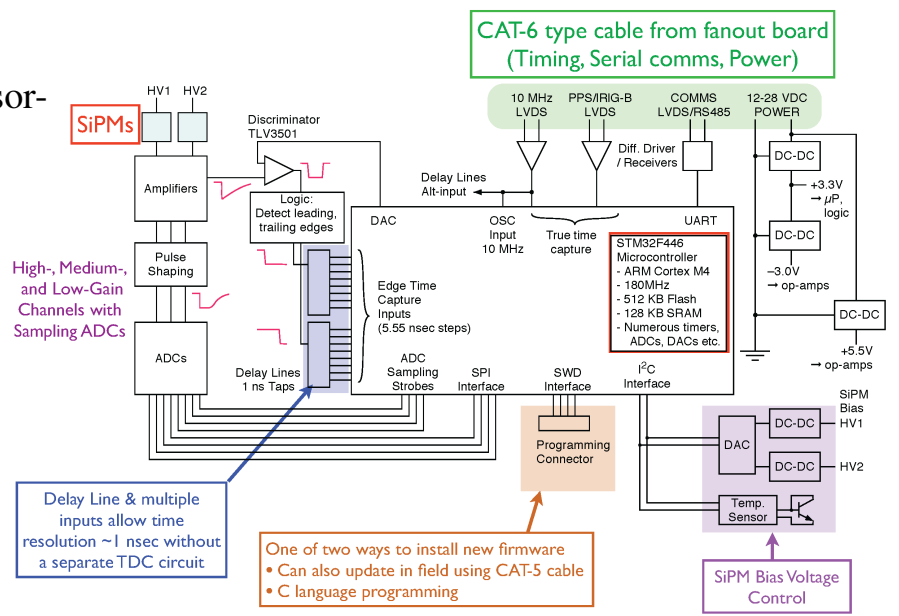

Figure 5: Layout of the $\mu \mathrm{DAQ}$ Board such that each input sees the same edge delayed by successive $\sim 1 \mathrm{~ns}$ increments before being recorded into "counter capture" registers that increment every $5.5 \mathrm{~ns}(180 \mathrm{MHz})$. Averaging multiple delayed inputs results in higher time resolution. The board also features amplifiers, with multiple gains for a wide dynamic range, after which the pulse is shaped for sample-and-hold ADCs from which charge is obtained versus time.

The $\mu \mathrm{DAQ}$ design enables digitizing the signal at the output of the SiPM before transmission to the SFH. At the SFH, a Linux single-board computer (Beagle Bone Black) polls the seven panels, each with their own $\mu \mathrm{DAQ}$, and then forwards data to the IceCube Laboratory over optical fiber. 


\section{The Scintillator Field Hub subsystem}

Communications, power, and timing for the scintillator array is accomplished via a network of Scintillator Field Hubs connected to backbone surface cabling to the IceCube Laboratory (ICL). Each SFH is the central DAQ node for a scintillator station (Fig. 2). The SFHs are synchronized to better than nanosecond precision by a White Rabbit (WR) Ethernet network [10], where the timing reference is a GPS receiver, and each SFH contains a WR node connected via single-mode optical fiber to a WR switch in the ICL. This link also provides gigabit Ethernet connectivity to each Scintillator Field Hub. The WR node in the SFH (WR-LEN ${ }^{4}$ ) provides timing and Ethernet connectivity to the DAQ via a copper Ethernet connection and two timing outputs: a reference 10 MHz clock, and a 1-PPS IRIG-B timestring. A separate copper cable supplies the power to each $\mathrm{SFH}$.

\section{Prototype detector efficiency in a Muon Tower}

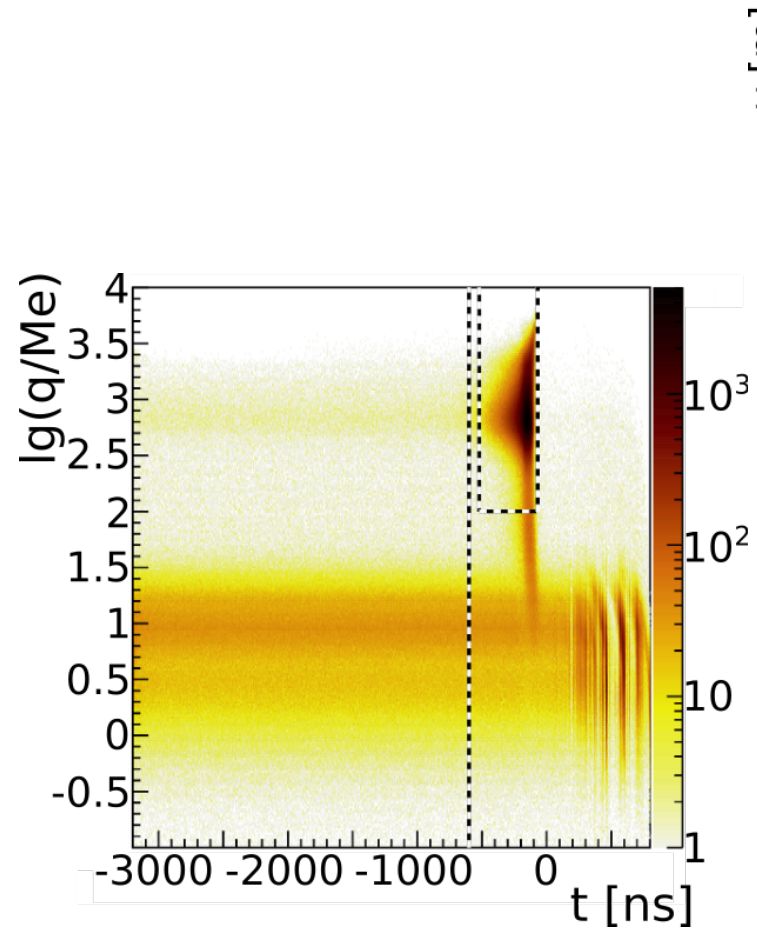

(a)

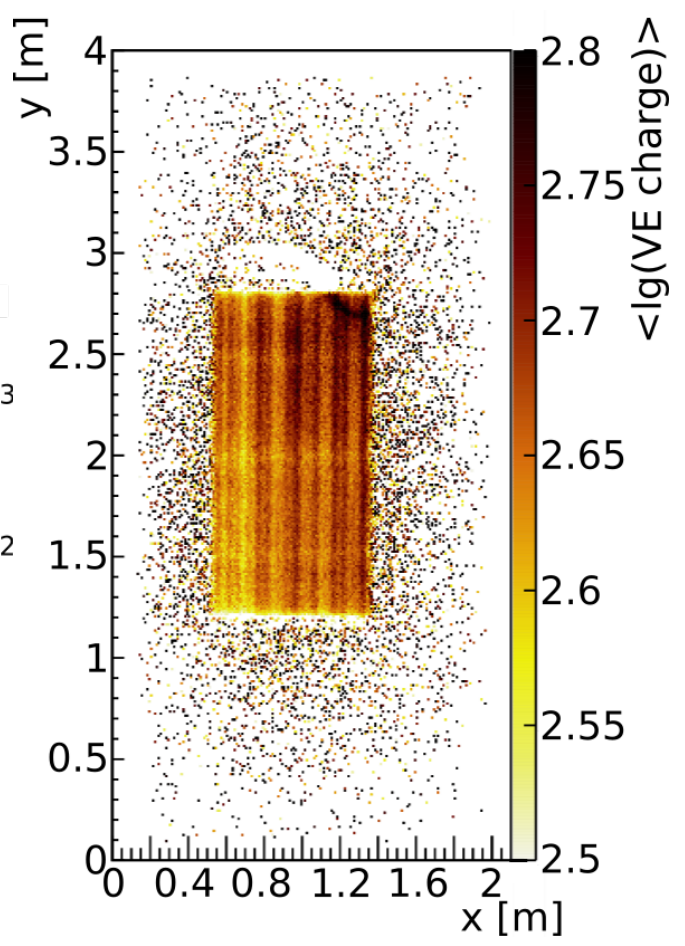

(b)

Figure 6: Left: Charge and time distribution of pulses detected with the prototype detector. The top region shows the high-charge band. The lower region the low-charge band and hence the SPE and the baseline. The dashed rectangle shows the quality cut for the MIPs. The distance between the high and low charge band shows the high light yield of a MIP compared to the base line. Right: Average pulse charge depending on the particle detection position. Single fibers are visible. Broken fibers or scintillators can be located and replaced. The higher average pulse charge located at $\mathrm{x}=1.2 \mathrm{~m} \mathrm{y}=2.8 \mathrm{~m}$ arises because the fiber bundle coupled to the analog readout module, was for prototyping purposes just placed at the surface of the scintillator bars.

\footnotetext{
${ }^{4}$ http://sevensols.com/index.php/products/wr-len/
} 
The Karlsruhe Institute of Technology (KIT) Muon Tower is a muon tracking detector with limited streamer tubes (LST) originally from the KASCADE experiment and presently used for calibration and efficiency measurements of scintillation detectors. The muon tower is capable of tracking muons with an angular resolution of better than $1^{\circ}$ and providing accurate position of muons for the test detectors. This enables obtaining single photoelectrons (SPEs) and the amount of SPEs per minimum ionizing particles (MIPs) in the test detector via a charge spectrum. Additionally, muon tomography is also possible. Due to the LST, the Muon Tower has an in-plane resolution of $1 \mathrm{~cm}^{2}$ that allows for correction of the zenith angle when calculating the average charge for vertical MIPs. It is also possible to investigate the efficiency and uniformity of the inner detector system (scintillator bars, routing of optical fibers, optical connection). The charge spectrum for through-going muons is shown in Fig. 6, left.

The plot in Fig. 6 (right) shows the average pulse charge depending on the particle position in a logarithmic scale. Due to the optimal positioning of the fibers to the photosensitive surface of the SiPM, the routing of the single fibers is visible. Due to this tomography-like feature of the muon tower, broken scintillators or fibers can be located and replaced.

\section{Summary \& Outlook}

We are currently developing a scintillator-based prototype detector as an upgrade to IceTop. Each detector comprises 7 panels based on scintillator bars read out with wavelength shifting fibers coupled to the photosensitive area of the SiPMs. We are investigating different options for fiber diameter and fiber-to-sensor coupling.

Currently, two versions of DAQs are being explored; one featuring an embedded system capable of capturing full waveforms and the other comprising a small microprocessor with timing and waveform capture with digital transmission of the data to the Beagle Bone Black based Field Hub. White Rabbit provides timing and communications to the individual detectors. We anticipate deploying a pair of prototype detectors in the 2017/18 Antarctic summer with an eventual phased deployment of an array of up to 37 stations planned in the future.

\section{References}

[1] IceCube Collaboration, M. G. Aartsen et al., J. Inst. 12 (2017) P03012.

[2] IceCube Collaboration, R. Abbasi et al., Nucl. Instr. Meth. A 700 (2013) 188 - 220.

[3] IceCube Collaboration, M. .G. Aartsen et al., PoS (ICRC2015) 628 (2015).

[4] K. Nitta et al., Nucl. Instr. Meth. A 535 (2004) 147.

[5] F. Retière et al. (T2K Collaboration), TIPP 2011 proceedings, Phys. Proc. 37 (2012) 1231 - 1240.

[6] MINERvA Collaboration, K. S. McFarland et al., Nucl. Phys. Proc. Suppl. 159 (2006) 107.

[7] Pierre Auger Collaboration, A. Aab et al., arXiv:1604.03637 (2016).

[8] E. Dietz-Laursonn et al., J. Inst. 12 (2017) 4P04026.

[9] T. Karg, A. Haungs, M. Kleifges et al., arXiv:1410.4685 (2014).

[10] P.P.M. Jansweijer, H.Z. Peek, and E. de Wolf, Nucl. Instr. Meth. A 725 (2013) 187 - 190. 